\title{
UMA VISÃO DE EDUCADORES QUE ACOMPANHAM ALUNOS COM TDAH: REVISÃO DE LITERATURA
}

\section{REVISÃO BIBLIOMÉTRICA}

PENHA JÚNIOR, Edson Ricardo Passos ${ }^{1}$

PENHA JÚNIOR, Edson Ricardo Passos. Uma visão de educadores que acompanham alunos com TDAH: Revisão de literatura. Revista Científica Multidisciplinar Núcleo do Conhecimento. Ano 05, Ed. 09, Vol. 09, pp. 46-55. Setembro de 2020.

ISSN: 2448-0959,

Link

de

acesso: https://www.nucleodoconhecimento.com.br/educacao/visao-de-educadores

\section{RESUMO}

Esse artigo tem por objetivo compreender a percepção dos professores acerca do Transtorno de Déficit de Atenção e Hiperatividade (TDAH), dentro do âmbito escolar, e suas práticas pedagógicas com os alunos envolvidos no processo no tempo de cada criança, nos seguintes seguimentos: "O aumento dos diagnósticos de TDAH nos últimos tempos", "A participação da escola e o TDH" e "Suspeita prematura do diagnóstico em crianças com TDAH ou a falta de diagnóstico especializado TDAH".A metodologia utilizada foi pesquisa bibliográfica partindo de materiais elaborados, principalmente de artigos científicos nos Bancos de Dados. Os resultados alcançados amparam a premissa elevada sobre os critérios metodológicos e diagnósticos, e a fonte de informação, da variabilidade das estimativas de prevalência a nível global.

Palavras-chave: TDAH, educador, diagnóstico escolar.

\section{INTRODUÇÃO}

Segundo o Manual Diagnostico e Estatístico de Transtornos Mentais (DSM-V) (2014) a característica essencial do TDAH, é o desafio e a hiperatividade-impulsividade, que

\footnotetext{
${ }^{1}$ Acadêmico de Psicologia da Faculdade Luciano Feijão.
} 
pode interferir no modo de funcionar e no modo de se desenvolver. A desatenção manifesta-se no comportamento, na divagação em tarefas, falta de persistência, dificuldade de manter o foco e desorganização, não constituindo consequências de desafios ou falta de compreensão, ou seja, para essas crianças fica um tanto cansativo visto que a maioria do sistema de ensino é homogênea.

Existem alguns comportamentos presentes nesse sentido que são muitas vezes estigmatizados pela sociedade, tais como: agitação, desobediência, dificuldades de aprendizagem, falta de concentração. Tais queixas sobre o comportamento passam muitas vezes pelos pais, muitos destes não possuem conhecimento sobre o diagnóstico e principalmente alguns professores, corroborando com os estigmas e preconceitos que cercam a criança com TDAH. Mendes (2010) afirma que, esse encontro de pretensões e de preocupação por parte da maioria dos docentes, no que diz respeito a formas de inclusão, sobre as divergências, diante da ausência de preparo ainda vem acontecendo.

Dessa forma, a escolha desse referido tema se deu devido a uma inquietude sobre como entender o processo de ensino-aprendizagem nestas crianças que são diagnosticadas com esse transtorno, ou seja, como em seu dia a dia essas crianças são vista por seus professores e como vivenciam seu entorno, isto é, compreender quais são as habilidades e/ou como uma criança com transtorno se desenvolve durante o processo de aprendizado no espaço escolar, envolvendo a preparação dos professores para acolher essa demanda.

Por isso, o objetivo principal deste estudo será compreender a percepção dos professores acerca do TDAH, dentro do âmbito escolar. Buscando ainda atingir os objetivos específicos: Investigar práticas pedagógicas com os alunos envolvidos no processo no tempo de cada criança. como os professores identificam crianças com sinais de TDAH; identificar quais fatores que levam o aluno à exclusão (familiar, escolar e social).

Justificando-se pelo fato que preciso entender que cada criança tem seu modo de aprender, seu modo de processamento das demandas impostas, já que seus 
processos de aprendizagem poderão ser de níveis diferentes das que não são portadoras do TDAH.

\section{METODOLOGIA}

A metodologia utilizada no presente projeto foi uma pesquisa exploratória tendo como objetivo proporcionar maior familiaridade com o problema contribuindo assim com hipótese e ainda compreender a percepção dos professores acerca do tema abordado.

Afirma-se que a metodologia bibliográfica é um procedimento importante para a produção de conhecimentos científicos, principalmente os poucos explorados, proporcionando suposições ou intepretações que servirão como base para outras pesquisas. (SASSO, 2007)

A pesquisa foi feita desde agosto até novembro de 2019 nos referidos bancos de dados: Scientific Electronic Library Online (Scielo), Periódicos Eletrônicos em Psicologia (PePSIC), Biblioteca Virtual em Saúde (BVS) e o Portal CAPES, são esses bancos de dados mais bem vistos e aceitos pela comunidade cientifica, onde por ventura foi realizada a escolha dos mesmos. Descritores: TDAH, escola, professor, infância, psicologia, família e desenvolvimento todos eles separados pelo operador booleano AND e agrupadas em pares de palavras.

Quanto aos procedimentos da pesquisa bibliográfica foram desenvolvidos a partir de materiais elaborados, principalmente de artigos científicos nos Bancos de dados: portal de Periódicos Eletrônicos de Psicologia (PePSIC) Biblioteca Virtual em Saúde (BVS), o Portal de Periódicos CAPES e Scientific Electronic Library Online (SciELO), amplamente difundidos e reconhecidos na comunidade acadêmica.

Logo após a leitura de tais bancos de dados, foram utilizados os critérios de inclusão: artigos originais dos últimos dez anos fizeram-se uma ligação com o tema abordado e artigos com idioma português. Entretanto, os artigos que não favorecem o tema ou artigos repetidos, foram excluídos. 


\section{RESULTADOS E DISCUSSÕES}

\subsection{O AUMENTO DOS DIAGNÓSTICOS DE TDAH NOS ÚLTIMOS TEMPOS}

Analisando o artigo de Caliman (2008) com o título "O TDAH: entre as funções, disfunções da atenção", mostrou que o TDAH vem sendo mostrado como um mau funcionamento na neuropsiquiátrica, que começa na infância e vai até a vida adulta, sendo um problema público, uma vez que é de uma perspectiva internacional. Contrário a isso, vem a sua adequação, sendo necessária uma coerência médica e biológica, na qual a narrativa defendida pela neuropsiquiatria mostra a existência de controvérsias que vem se consolidando. Este artigo teve objetivo principal de analisar os termos delicados que estabelecem as funções e disfunções desse transtorno.

No artigo de Tassotti (2015) com o título "TDAH: diagnóstico diferencial e tratamento" vê-se que, esse assunto vem ganhando destaque nos últimos anos, tanto para os pais, como professores e profissionais de Saúde Mental, até mesmo considerando ele como principal distúrbio psicológico na juventude. Acometendo especialmente as crianças e os adolescentes de idade escolar. Ele mostrou que vários estudos assinalam sobre as possíveis causas genéticas e ambientais, afirmando até mesmo que não tem uma causa exclusiva que resulte no TDAH. Tais comportamentos, hora normais na vida criança, acabam sendo vistos como comportamentos patológicos. Mostrou ainda a importância do diagnóstico diferencial que o TDAH tem, assim, facilmente confundido com outros distúrbios, muitas vezes acompanhado de comorbidades. Ele salientou por fim que a prática da terapêutica medicamentosa só terá resultados aceitáveis se tiver um acompanhado psicológico, por parte do ambiente fundamenta a família.

Observa-se no artigo que Souza et. al. (2017), intitulado "Dificuldades no diagnóstico de TDAH em crianças", que esse transtorno é uma doença que tem maior prevalência nas crianças em idade escolar. Entretanto, muitos entendem que tem um diagnóstico de baixa morbidade, mesmo o TDAH sendo reconhecido ultimamente como algo 
importante, não apenas pelo impacto ativo e social, mas pela alta prevalência em comorbidades psiquiátricas. Assim, os déficits cognitivos globais e transtornos invasivos sobre o aumento de transtornos de aprendizado, nas quais as condições complexas associadas aos sintomas de TDAH têm suas representações agravadas, promovendo uma maior atenção e táticas terapêuticas mais individualizadas.

\subsection{PARTICIPAÇÃO DA ESCOLA E O TDAH}

Observou-se na visão desses três autores que, outros tipos de transtornos, como por exemplo, o Transtorno Opositivo Desafiador (TOD), faz com que, como morbidade, ele piore o prognóstico do TDAH. Com tudo, o TDAH é um mau funcionamento neuropsiquiátrico como também visto como um problema de ordem pública de uma visão internacional. E essas crianças continuam tendo essa doença na vida adulta, já que de ordem genética e biológica, ou seja, está ligada ao cérebro.

O TDAH é considerado como uma doença porque traz problemas na vida desse indivíduo, que não conseguem executar tarefas como a maioria das pessoas. E justamente por estarmos inseridos em uma sociedade que cobra uma produtividade maior, essas pessoas com o transtorno aparecem mais, ou seja, os diagnósticos têm crescidos nos últimos anos.

No artigo de Reis; De Camargo (2008) que trata sobre "Práticas escolares e desempenho acadêmico de alunos com TDAH", foi mostrado como os cursos de formação de professores no Ensino Superior, trabalham sob as suas variadas dimensões. O levantamento focou em cinco adultos que foram diagnosticados com TDAH. Foi visto que os resultados obtidos podem contribuir expressivamente para que os novos professores possam conhecer sobre os pontos determinantes na execução escolar de alunos com o transtorno, bem como guiar na busca da sociedade com outros profissionais quando esta se fizer necessária.

Rocha; Del Prette (2017) no ensaio "Habilidades sociais educativas para mães de crianças com TDAH e a inclusão escolar" apresentam uma categoria de Educação Especial, focando nas implicações do transtorno de TDAH, no ambiente escolar e qual 
era o papel dos pais dentro da parceria família-escola. Destacando sobre as habilidades pessoais e parentais daqueles diretamente envolvidos com crianças diagnosticadas com TDAH, apresentando a ações educativas mais efetivas, que buscavam cooperar com a ascensão do incremento das desenvolturas sociais e acadêmicas, aprovando que mostrem as oportunidades sociais e educacionais no ambiente escolar.

Resende (2009), com o artigo intitulado "Alunos do ensino fundamental com sintomas de TDAH: A motivação escolar com a prática de atividades lúdico-recreativas baseadas na pedagogia do judô", mostrou que o TDAH afeta entre 3 a $7 \%$ das crianças em idade escolar mundialmente, ou seja, o número passa dos dois milhões de alunos. Como se trata de um transtorno neurobiológico caracterizado pela dificuldade em manter a atenção, a hiperatividade, o excesso de movimento e a impulsividade/dificuldades para controlar os impulsos, acaba se manifestando com mais frequência na vida acadêmica, mas pode ser encontrado também na vida familiar, pessoal e social. Sendo que a atenção pedagógica constitui um grande desafio para os educadores, já que a demanda desses agentes ações diferenciadas levando em conta o desenvolvimento de todas as personalidades.

Observou-se que ainda existe um despreparo por parte dos professores, com relação aos alunos com TDAH. Os autores dos três artigos mostraram também a importância da família para com a criança com TDAH, dentro de sala de aula. Função essa que, de certa forma, coloca a família como principal elemento e o mais fundamental na educação, seja dentro de sala de aula, ou mesmo na família, e a importância da união com a escola para combater esses desafios postos ao desenvolvimento e a aprendizagem da criança. Mostrando assim que existem formas diferentes de âmbitos diferentes para se trabalharem em uma causa especifica que diz respeito ao TDAH.

Além disso, percebe-se que muito ainda precisa fazer sobre os pontos que norteiam o ensino de crianças com TDAH, onde se pôde verificar cada contrapondo das leituras o papel do educador, quando confrontado com uma criança que tenha sido diagnosticada com esse transtorno, ou mesmo que ela apenas apresente uma hiperatividade diferenciada, mostrando a importância da diversificação das aulas 
ministradas, e salientando sobre a necessidade de se trabalhar com todo grupo de apoio, sejam eles pais, professores, escola, psicólogos ou profissionais da área de saúde.

O presente estudo apresentou limitações uma vez que a base de dados do levantamento eram artigos, e tem-se uma defasagem grande sobre temas diretamente relacionados a área educacional das crianças. É preciso entender mais sobre as prevalências das principais comorbidades do TDAH, e ser mais averiguado sobre esses estudos categorizados.

Os resultados obtidos defendem o princípio de que procedimentos metodológicos e diagnósticos, seleção de amostras, e a fonte de informação, expandem globalmente a diversidade das estimativas de prevalência. Pesquisas futuramente realizadas poderão analisar a interrelação desses coeficientes no estabelecimento do diagnóstico do TDAH e como conseguinte a taxa de prevalência alcançada.

\subsection{SUSPEITA PREMATURA DO DIAGNÓSTICO EM CRIANÇAS COM TDAH OU A FALTA DE DIAGNÓSTICO DO TDAH}

O trabalho de Sensu; Barbosa (2011)com a temática sobre "A Prática Docente Frente a Suspeita e Diagnóstico do Transtorno de Déficit e Hiperatividade de Crianças em Fase Escolar", trata sobre este transtorno complexo, que arremete crianças em idade escolar, buscando mostrar o processo que a criança passa e como é relevante para seu desenvolvimento, pois, está ligado diretamente ao seu aprendizado e desenvolvimento cognitivo. As crianças que apresentam tais sintomas mostram diretamente em seu comportamento e processo de ensino-aprendizagem a diferença. Mostrou também o importante papel da escola na compreensão dos comportamentos e no delineamento de um programa de intervenção pedagógica, no qual a função seja inserir tais crianças no contexto escolar, consentindo o avanço no aprendizado e desenvolvimento intelectual, além de proporcionar condições que permitam a metodologia de interação social e o sucesso no rendimento escolar. 
Já o trabalho de Peixoto e Rodrigues (2008), "Diagnóstico e tratamento de TDAH em crianças escolares, segundo profissionais da saúde mental", teve o desígnio de levantar quais eram os critérios de diagnóstico e tratamento realizados em crianças em idade escolar, que apresentaram TDAH, mostrando como profissionais da área de saúde mental atuavam nos consultórios privados, na região da Grande Vitória/ES. Conseguiram mostrar todas as informações obtidas através da sua pesquisa, além de indicar critérios pela literatura especializada. Mostrou que a maioria dos profissionais indicava medicações como forma principal no tratamento desse transtorno, não sem ter uma equipe multidisciplinar, a despeito do reconhecimento da necessidade de ajuste sobre a intervenção medicamentosa de outras modalidades, principalmente a psicoterápica.

Já no trabalho de Santos (2013), "O diagnóstico do TDAH: concepções de professoras de atendimento especializado, outros profissionais da educação e profissionais da saúde", discutiam a circulação e assimilação sobre os diagnósticos de TDAH, levando em conta os cenários escolares. Abordou o contexto de uma mudança de paradigma, na qual as ciências humanas permitiram ser referenciadas para as diversas interfaces da neurociência. Levaram em consideração as diversas questões referentes ao diagnóstico do TDAH, no âmbito escolar, sendo mostradas as opiniões positivas e negativas, explicando principalmente a positividade relacionada com o oferecimento dos serviços de apoio à aprendizagem nas organizações de estratégias individuais, incluindo a criança nas atividades e expondo sobre a negatividade pautada na construção de rótulos e estigmas. Ao final foi realizada a análise dos artigos selecionados para a pesquisa e serão divididos em três categorias de análise (conceituais analíticas teóricas e empíricas).

Segundo o autor Bardin (2011) estabelece uma explanação analítica indicando as possíveis utilizações da análise de conteúdo como um método de categorias que autorizam a classificação dos integrantes do conceito da mensagem em espécie de gavetas. Essa análise de conteúdo é também uma análise de significados, contra isso, esta seria de uma descrição sistemática, quantitativa e objetiva do conteúdo removido das comunicações e sua correspondente interpretação. 
Como citado anteriormente, foi visto que o Transtorno de Déficit de Atenção e Hiperatividade não é uma patologia que surgiu recentemente, o interesse por essa temática vem desde o século XIX. Porém, no Brasil ainda os estudos levantados sobre o assunto ainda são muito desanimadores e raramente se tem algo a respeito da educação e acompanhamento da criança dentro do ambiente escolar. É corriqueiro professores apontarem os pais como os culpados por não darem limites aos seus filhos, gerando culpa na família, que mesmo sendo responsável direta pela criança, não convive tanto com a criança quanto os educadores, durante os anos escolares.

Muitas crianças são rotuladas, pelas escolas, de mal-educadas, rebeldes. Assim sendo, analisar até onde vão os estudos dessa temática são importantes, pois é com essa informação, correta sobre o TDAH, que pode-se fazer uma correta orientação com pais e professores, uma vez que eles são uma ferramenta muito importante para fazer o correto diagnóstico, assim como também para a concretização e o sucesso, ou não, da terapêutica.

\section{CONSIDERAÇÕES FINAIS}

Hoje em dia as relações que dizem respeito a família para com as crianças com TDAH fazem uma espécie de estigma voltado para escola, ou seja, basta a criança começar a apresentar comportamentos mais desatentos, mais inquietos, e começar a interferir no desempenho dela na escola, que acaba sendo a principal preocupação dos pais e dos professores por colocarem como principal hipótese do problema algum transtorno, dessa forma, o TDAH aparece como uma "explicação" do comportamento da criança.

O maior problema visto dentro desse levantamento foram às distinções entre as funções e disfunções relacionadas à atenção e à otimização do diagnóstico do TDAH. Referindo-se sobre as estratégias pedagógicas voltadas para superação dos obstáculos vistos hoje. O diagnóstico do TDAH vem através do estabelecido no interior deste campo híbrido, no qual a análise do TDAH como um diagnóstico desafiante, onde é necessária uma junta de profissionais para ser avaliado a neurótica, no diagnóstico e terapêutico. 
Tentando deixar de lado a possibilidade de que a criança tenha o transtorno, é preciso explorar outras probabilidades antes de dar um diagnóstico psiquiátrico precoce errado e assim aplicar medicações em uma criança que não tenha TDAH. Pois o que mais surgiu durante a pesquisa foi o fato que a hipótese de uma criança ter TDAH, acaba sendo a primeira coisa que vem à mente de muitos pais, educadores ou mesmo da escola. Isso tem ocasionado um aumento exponencial da taxa de "crianças com TDAH", por acabar sendo um esclarecimento mais simples e instantâneo de respostas.

Por fim, viu-se que é incontestável a necessidade de serem feitas mais pesquisa no âmbito metodológico, buscando averiguar a proporção das diversificadas estimativas desse transtorno, uma vez que cria dificuldade diretamente sobre as políticas públicas voltadas a educação da população, para que dessa forma os educadores possuam mais conhecimento sobre possíveis casos de TDAH e como esse transtorno ocorre com a criança, sendo assim capaz de ser mediador da educação para a criança diagnóstica.

\section{REFERÊNCIAS}

BARDIN, L. (2011). Análise de conteúdo. São Paulo: Edições 70.

CALIMAN, LV. A biologia moral da atenção: a constituição do sujeito desatento. Rio de Janeiro (RJ): Programa de Pós-Graduação em Saúde Coletiva, Instituto de Medicina Social, Universidade do Estado do Rio de Janeiro; 2006.

DSM-V. AMERICAN PSYCHIATRIC ASSOCIATION et al. DSM-5: Manual diagnóstico e estatístico de transtornos mentais. Artmed Editora, 201.

MENDES, PDE Adriana Valéria Fantin. TDAH, O DESAFIO DA INCLUSÃO.

PEIXOTO, A. L; RODRIGUES, M. Diagnóstico e tratamento de TDAH em crianças escolares, segundo profissionais da saúde mental. Aletheia, n. 28, p. 91-103, 2008. Disponível em: https://www.redalyc.org/pdf/1150/115012542008.pdf. Acesso em: 22 nov. 2019. 
REIS, M.; DE CAMARGO, D. Práticas escolares e desempenho acadêmico de alunos com TDAH. Psicologia escolar e educacional, v. 12, n. 1, p. 89-100, 2008. Disponível em: https://www.redalyc.org/pdf/2823/282321824007.pdf. Acesso em: 24 nov. 2019

RESENDE, FAH. Alunos do ensino fundamental com sintomas de TDAH: A motivação escolar com a prática de atividades lúdico-recreativas baseadas na pedagogia do judô. EDUCERE, p. 9143-9158, 2009.

ROCHA, M. M.; DEL PRETTE, Z. A. P. Habilidades sociais educativas para mães de crianças com TDAH e a inclusão escolar. Psicologia Argumento, v. 28, n. 60, 2017.Disponível

em: https://periodicos.pucpr.br/index.php/psicologiaargumento/article /view /19723/19 047. Acesso em: 23 nov. 2019

SANTOS, K. O diagnóstico do TDAH: concepções de professoras de atendimento especializado, outros profissionais da educação e profissionais da saúde. 2013. Disponível em: http://www.repositorio.unifesp.br/handle/11600/48312. Acesso em: 22 nov. 2019

SASSO DE LIMA, T. C.; TAMASO MIOTO, R. C. Procedimentos metodológicos na construção do conhecimento científico: a pesquisa bibliográfica. Revista Katálysis, v. 10, 2007.

SENSU, PÓSGEME; BARBOSA, CLAUDIA WALTRICK MACHADO. A PRÁTICA DOCENTE FRENTE A SUSPEITA E DIAGNÓSTICO DO TRANSTORNO DE DÉFICIT DE ATENÇÃO E HIPERATIVIDADE (TDAH) DE CRIANÇAS EM FASE ESCOLAR.

SOUSA, A. C. et al. Como a Família e a Escola Podem Contribuir Para o Processo de Aprendizagem de Alunos Portadores de TDAH. ANAIS 4" JORNADA DE INICIAÇÃO CIENTÍFICA, p. 99, 201 
TASSOTTI, C. TDAH: diagnóstico diferencial e tratamento. 2015. Disponível em: http://bibliodigital.unijui.edu.br:8080/xmlui/bitstream/handle/123456789/3051/TCC\%2 0corrigido.\%20.pdf?sequence=1. Acesso em: 20 nov. 2019

Enviado: Agosto, 2020.

Aprovado: Setembro, 2020. 